E17-2016-8

N. N. Bogolubov (Jr. $)^{1,2, *}$, A. V. Soldatov ${ }^{1, * *}$

\title{
VARIATIONAL MASTER EQUATION APPROACH TO DYNAMICS OF MAGNETIC MOMENTS
}

This report was presented at a seminar at the Bogoliubov Laboratory of Theoretical Physics, JINR, Dubna

Submitted to «Particles and Nuclei, Letters»

\footnotetext{
${ }^{1}$ V. A. Steklov Mathematical Institute of the Russian Academy of Sciences, 8 Gubkin Str., 119991 Moscow, Russia

${ }^{2}$ Joint Institute for Nuclear Research, Dubna

*E-mail: nikolai_bogolubov@ hotmail.com

**E-mail: soldatov@mi.ras.ru
} 
Боголюбов Н. Н. (мл.), Солдатов А. В.

Метод вариационного управляющего уравнения в динамике

магнитных моментов

Неравновесные свойства модельной системы, состоящей из подсистемы магнитных моментов, сильно взаимодействующих с выделенной модой бозонного поля и слабо взаимодействующих с бозонным термостатом, исследованы посредством подхода, сочетающего в себе черты метода неравновесного управляющего уравнения (master equation) и метода аппроксимирующего гамильтониана. Полученное вариационное управляющее уравнение допускает численный анализ и может быть использовано для получения системы обыкновенных дифференциальных уравнений для физических переменных, относящихся к подсистеме магнитных моментов. В результате анализа полученного вариационного управляющего уравнения выявлено, что макроскопическое заполнение выделенной полевой бозе-моды при достаточно низкой температуре оказывает влияние на релаксационную динамику магнитных моментов.

Работа выполнена в Математическом институте им. В. А. Стеклова Российской академии наук.

Препринт Объединенного института ядерных исследований. Дубна, 2016

Bogolubov N. N. (Jr.), Soldatov A. V.

E17-2016-8

Variational Master Equation Approach to Dynamics of Magnetic Moments

Non-equilibrium properties of a model system comprised of a subsystem of magnetic moments strongly coupled to a selected Bose field mode and weakly coupled to a heat bath made of a plurality of Bose field modes were studied on the basis of non-equilibrium master equation approach combined with the approximating Hamiltonian method. A variational master equation derived within this approach is tractable numerically and can be readily used to derive a set of ordinary differential equations for various relevant physical variables belonging to the subsystem of magnetic moments. Upon further analysis of the thus obtained variational master equation, an influence of the macroscopic filling of the selected Bose field mode at low enough temperatures on the relaxation dynamics of magnetic moments was revealed.

The investigation has been performed at the V. A. Steklov Mathematical Institute of the Russian Academy of Sciences. 


\section{INTRODUCTION}

An approximating Hamiltonian method [1] proved efficient when it comes to study in a mathematically rigorous way the thermodynamic properties of several broad enough classes of quantum model systems. It is based on the idea that in some cases the original model Hamiltonian of a many-body system can be simplified by replacing some operator complexes, standing for intensive variables, by complex numbers ( $C$-numbers). As a result, one arrives at the exactly solvable Hamiltonian in the sense that the thermodynamically equilibrium free energy or free energy density, associated with this Hamiltonian, can be derived analytically as a function of the temperature, all the model parameters entering the Hamiltonian in question, and the above-mentioned $c$-numbers. For some classes of physically relevant models it was shown possible to choose the values of these $c$-numbers in such a way that the thermodynamic behavior of this simplified Hamiltonian becomes totally equivalent to the one of the original Hamiltonian, in which case the simplified Hamiltonian is called the approximating Hamiltonian. By construction, all the thermodynamic properties of the approximating Hamiltonian coincide precisely with the properties spawned by the initial quantum model Hamiltonian. As a consequence, collective phenomena in many-body systems, such as, for example, thermodynamically equilibrium phase transitions, can be studied rigorously within this approach. The purpose of the present contribution is to show that approximating Hamiltonians are useful not only in studies of thermodynamically equilibrium properties but may give insight into their nonequilibrium behavior too.

\section{MODEL HAMILTONIAN}

The model under consideration is represented by the following Hamiltonian:

$$
H=H_{0}(B)+H_{0}(C)+H_{0}(S)+H_{B S}+H_{C S}
$$

where

$$
H_{0}(B)=\omega b^{+} b, \quad H_{0}(C)=\sum_{i=1}^{N} \sum_{\mathbf{k}} \omega_{i, \mathbf{k}} c_{i, \mathbf{k}}^{+} c_{i, \mathbf{k}}, \quad H_{0}(S)=\epsilon S^{z}=\epsilon \sum_{i=1}^{N} s_{i}^{z}
$$




$$
\begin{gathered}
H_{B S}=\frac{\lambda}{\sqrt{V}}\left(b S^{+}+b^{+} S^{-}\right)=\frac{\lambda}{\sqrt{V}}\left(b \sum_{i=1}^{N} s_{i}^{+}+b^{+} \sum_{i=1}^{N} s_{i}^{-}\right), \\
H_{C S}=\sum_{i=1}^{N} \sum_{\mathbf{k}} g_{i, \mathbf{k}}\left(c_{i, \mathbf{k}} s_{i}^{+}+c_{i, \mathbf{k}}^{+} s_{i}^{-}\right),
\end{gathered}
$$

and

$$
S^{z}=\sum_{i=1}^{N} s_{i}^{z}, \quad S^{ \pm}=\sum_{i=1}^{N} s_{i}^{ \pm}, \quad s_{i}^{z}=\frac{1}{2} \sigma_{i}^{z}, \quad s_{i}^{ \pm}=\sigma_{i}^{ \pm}=\frac{1}{2}\left(\sigma_{i}^{x} \pm i \sigma_{i}^{y}\right),
$$

with the Pauli matrices $\sigma_{i}^{z}, \sigma_{i}^{x}$ and $\sigma_{i}^{y}$. The operators $c_{i, \mathbf{k}}^{+}, c_{i, \mathbf{k}}$ and $b^{+}, b$ are the Bose operators obeying usual commutation rules

$$
\begin{gathered}
{\left[c_{i, \mathbf{k}}, c_{i, \mathbf{k}^{\prime}}^{+}\right]=\delta_{i, j} \delta_{\mathbf{k}, \mathbf{k}^{\prime}}, \quad\left[c_{i, \mathbf{k}}, c_{i, \mathbf{k}^{\prime}}\right]=0, \quad\left[b, b^{+}\right]=1,} \\
{[b, b]=0, \quad\left[b, c_{i, \mathbf{k}}\right]=0, \quad\left[b^{+}, c_{i, \mathbf{k}}\right]=0 .}
\end{gathered}
$$

So, the Hamiltonian (4) can be interpreted in terms of a subsystem $S$ of $N$ twolevel quantum objects, for example, magnetic moments, contained in a cavity of volume $V$, strongly interacting with some selected Bose-mode $b$ and, at the same time, weakly interacting with a plurality of Bose modes $c_{i, \mathbf{k}}$ comprising a heat bath.

\section{BASICS OF THE APPROXIMATING HAMILTONIAN METHOD}

Let us illustrate the workings of the approximating Hamiltonian method by considering a model Hamiltonian $(\hbar=1)$

$$
H=\omega b^{+} b+\epsilon S^{z}+\frac{\lambda}{\sqrt{V}}\left(b S^{+}+b^{+} S^{-}\right),
$$

which is actually a part

$$
H=H_{0}(B)+H_{0}(S)+H_{B S}
$$

of the total Hamiltonian (4) under consideration. Let us rewrite Eq. (4) by adding infinitesimal symmetry breaking sources $\nu, \nu^{*}$ :

$$
H(\nu)=\omega b^{+} b+H_{S}+\frac{\lambda}{\sqrt{V}}\left(b S^{+}+b^{+} S^{-}\right)-\sqrt{V} \lambda\left(b \nu^{*}+b^{+} \nu\right)
$$

and build a form for the approximating Hamiltonian $H_{a}(\nu, \eta)$ :

$$
H(\nu)=H_{a}(\nu, \eta)+H_{I B S}(\nu, \eta)
$$




$$
\begin{gathered}
H_{a}(\nu, \eta)=H_{a}(B, \eta, \nu)+H_{a}(S, \eta, \nu)= \\
=H_{0}(S)+V \omega\left(\frac{b^{+}}{\sqrt{V}}-\frac{\nu^{*}}{\omega}+\frac{\lambda}{\omega} \eta^{*}\right)\left(\frac{b}{\sqrt{V}}-\frac{\nu}{\omega}+\frac{\lambda}{\omega} \eta\right)- \\
-V\left\{\frac{\lambda^{2}}{\omega}\left(\eta \frac{S^{+}}{V}+\eta^{*} \frac{S^{-}}{V}\right)-\frac{\lambda^{2}}{\omega}|\eta|^{2}-\frac{\lambda}{\omega}\left(\nu^{*} \frac{S^{-}}{V}+\nu \frac{S^{+}}{V}\right)+\frac{|\nu|^{2}}{\omega}\right\}, \\
H_{I B S}(\nu, \eta)=V \lambda\left\{\left(\frac{b^{+}}{\sqrt{V}}-\frac{\nu^{*}}{\omega}+\frac{\lambda}{\omega} \eta^{*}\right)\left(\frac{S^{-}}{V}-\eta\right)+\text { h.c. }\right\},
\end{gathered}
$$

where $\eta$ is a complex parameter. It can be easily proved that

$$
\begin{aligned}
& -\left\langle\frac{b}{\sqrt{V}}\right\rangle_{H(\nu)}=\frac{\lambda}{\omega}\left\langle\frac{S^{-}}{V}\right\rangle_{H(\nu)}-\frac{\nu}{\omega}, \\
& -\left\langle\frac{b^{+}}{\sqrt{V}}\right\rangle_{H(\nu)}=\frac{\lambda}{\omega}\left\langle\frac{S^{+}}{V}\right\rangle_{H(\nu)}-\frac{\nu^{*}}{\omega},
\end{aligned}
$$

where

$$
\langle\ldots\rangle_{H}=\frac{\operatorname{Tr}\left(\ldots \mathrm{e}^{-\beta H}\right)}{\operatorname{Tr}\left(\mathrm{e}^{-\beta H}\right)} .
$$

It is seen from (8) that parameters $\eta$ and $\frac{\lambda}{\omega} \eta$ stand for the operators $S^{-} / V$ and $b / \sqrt{V}$ respectively as the correspondent $c$-numbers. It was proved in [1] that serial inequalities hold for models of the type (4)

$$
\begin{aligned}
& \quad \lambda\left\{\left\langle\left(\frac{b^{+}}{\sqrt{V}}-\left\langle\frac{b^{+}}{\sqrt{V}}\right\rangle_{H_{a}(\nu, \eta)}\right)\left(\frac{S^{-}}{V}-\left\langle\frac{S^{-}}{V}\right\rangle_{H_{a}(\nu, \eta)}\right)+\text { h.c. }\right\rangle_{H_{a}(\nu, \eta)}\right\} \leq \\
& \leq f_{V}\left[H_{a}(\nu, \eta)\right]-f_{V}[H(\nu)] \leq f_{V}\left[H_{a}\left(\nu,\left\langle\left\{\frac{S^{ \pm}}{V}\right\}\right\rangle_{H(\nu)}\right)\right]-f_{V}[H(\nu)] \leq \\
& \leq-\lambda\left\{\left\langle\left(\frac{b^{+}}{\sqrt{V}}-\left\langle\frac{b^{+}}{\sqrt{V}}\right\rangle_{H(\nu)}\right)\left(\frac{S^{-}}{V}-\left\langle\frac{S^{-}}{V}\right\rangle_{H(\nu)}\right)+\text { h.c. }\right\rangle_{H(\nu)}\right\},
\end{aligned}
$$

where

$$
f_{V}[H]=-\frac{1}{\beta V} \ln \left(\operatorname{Tr}\left(\mathrm{e}^{-\beta H}\right)\right)
$$

is the free energy density. 
Therefore,

$$
\begin{gathered}
0 \leq \min _{\{\eta\}} f_{V}\left[H_{a}(\nu, \eta)\right]-f_{V}[H(\nu)] \leq \\
\leq f_{V}\left[H_{a}\left(\nu,\left\langle\left\{\frac{S^{ \pm}}{V}\right\}\right\rangle_{H(\nu)}\right)\right]-f_{V}[H(\nu)] \leq \\
\leq-\sum_{i=1}^{M} \lambda\left\{\left\langle\left(\frac{b^{+}}{\sqrt{V}}-\left\langle\frac{b^{+}}{\sqrt{V}}\right\rangle_{H(\nu)}\right)\left(\frac{S^{-}}{V}-\left\langle\frac{S^{-}}{V}\right\rangle_{H(\nu)}\right)+\text { h.c. }\right\rangle_{H(\nu)}\right\} .
\end{gathered}
$$

Here

$$
\begin{gathered}
f_{V}\left[H_{a}(\eta)\right]=\frac{1}{\beta V} \ln \left(1-\mathrm{e}^{-\beta \omega}\right)-\frac{N}{\beta V} \ln \left(2 \cosh \left(\frac{1}{2} E\left(\left\{\eta, \eta^{*}\right\}\right)\right)\right)+\frac{\lambda^{2}}{\omega}|\eta|^{2} \\
E\left(\left\{\eta, \eta^{*}\right\}\right)=\left\{\epsilon^{2}+4\left[\frac{\lambda^{2}}{\omega} \operatorname{Re}(\eta)\right]^{2}+4\left[\frac{\lambda^{2}}{\omega} \operatorname{Im}(\eta)\right]^{2}\right\}^{1 / 2}
\end{gathered}
$$

and the absolute minimum for the density of the free energy $f_{V}\left[H_{a}(\nu, \eta)\right]$ is to be searched for over the whole range of parameter $\eta$ and is provided by solution of the following equation:

$$
\eta=\frac{1}{E(|\eta|)} \frac{\lambda^{2}}{\omega} \eta \tanh \left(\frac{\beta E(|\eta|)}{2}\right) .
$$

Equation (18) has non-trivial solution if

$$
\frac{2}{|\epsilon|} \frac{\lambda^{2}}{\omega}>1
$$

and this non-trivial solution provides the absolute minimum to (16) if the temperature $\theta=1 / \beta=k T$ is smaller than the critical temperature $\theta_{c}$ :

$$
\theta<\theta_{c}=\frac{1}{4}|\epsilon|\left\{\operatorname{Arth}\left(\frac{\omega \epsilon}{2 \lambda^{2}}\right)\right\}^{-1} .
$$

It was proved [1] that in the so-called thermodynamic limit (t-lim): $N \rightarrow \infty$, $V \rightarrow \infty, N / V=1 / v=$ const, the absolute minimum of the free energy density (16) for the approximating Hamiltonian equals the free energy density for the original model for the whole range of model parameters (4). 


\section{TIME-CONVOLUTIONLESS MASTER EQUATION}

As usual, the complete dynamics of the whole system $S+B+C$ is governed by the Liouville-von Neumann equation for the density matrix $\rho(t)$

$$
\frac{\partial}{\partial t} \rho(t)=-i\left[H_{0}(\eta)+H_{I}(\eta), \rho(t)\right], \quad \operatorname{Tr}_{S, B, C}(\rho(t))=1,
$$

where

$$
H_{0}=H_{a}(\eta)+H_{0}(C), \quad H_{I}(\eta)=H_{I S B}(\eta)+H_{S C},
$$

or, in the interaction picture

$$
\begin{gathered}
\tilde{\rho}(t)=\mathrm{e}^{i H_{0} t} \rho(t) \mathrm{e}^{-i H_{0} t}=\mathrm{e}^{i H_{a} t} \mathrm{e}^{i H_{0}(C) t} \rho(t) \mathrm{e}^{-i H_{0}(C) t} \mathrm{e}^{-i H_{a} t}, \\
\tilde{H}_{I}(t)=\mathrm{e}^{i H_{0} t} H_{I} \mathrm{e}^{-i H_{0} t}=\mathrm{e}^{i H_{a} t} \mathrm{e}^{i H_{0}(C) t} H_{I}(\eta) \mathrm{e}^{-i H_{0}(C) t} \mathrm{e}^{-i H_{a}\left(t-t_{0}\right)}, \\
\frac{\partial}{\partial t} \tilde{\rho}(t)=-i\left[\tilde{H}_{I}(t), \tilde{\rho}(t)\right] .
\end{gathered}
$$

To study the explicit dynamics of open quantum subsystem $S$, we take recourse to the master equation methods as being more universal and numerically tractable. In our particular case, this technique provides an equation of motion for the reduced density matrix of the subsystem $S$ only, $\rho_{S}(t)=\operatorname{Tr}_{B, C}(\rho(t))$, without any necessity to account for the behavior of the environment represented by the subsystems $B$ and $C$. There are several ways to derive master equations for $\rho_{S}(t)$, and any such method provides formally exact closed master equation. Unfortunately, none of these equations allow either for explicit analytical solution or for direct numerical analysis in the general case. Thus, all these equations only serve as a starting point for derivation of much more tractable approximate equations. And, as a rule, any such approximation includes some kind of perturbative expansion in a small parameter, such as the system-environment coupling strength, par excellence. Normally, this expansion is truncated at the second order. However, this practice relies on the system-environment coupling in the initial Hamiltonian being small, which is not always the case. Moreover, this approach totally ignores the influence of temperature on the subsystem evolution; i.e., it does not account for possible occurrence of collective effects. Below we will show that the approximating Hamiltonian approach may help ameliorate these drawbacks to some extent.

When choosing between different varieties of master equation, we would prefer to derive here the so-called time-local master equation [2], also known as time-convolutionless master equation. This equation does not contain any timeconvolution kernel and, as a consequence, can be solved numerically relatively 
easy. At the second truncation order this time-convolutionless master equation reads as

$$
\frac{\partial}{\partial t} \tilde{\rho}_{S}(t)=-\int_{t_{0}}^{t} d s \operatorname{Tr}_{B}\left[\tilde{H}_{I}(t),\left[\tilde{H}_{I}(s), \tilde{\rho}_{S}(t) \rho_{B C}\left(t_{0}\right)\right]\right],
$$

where

$$
\tilde{\rho}_{S}(t)=\operatorname{Tr}_{B, C}(\tilde{\rho}(t)), \quad \tilde{\rho}_{B C}\left(t_{0}\right)=\operatorname{Tr}_{S}\left(\tilde{\rho}\left(t_{0}\right)\right),
$$

and, as usual, we chose separable initial condition for the full density matrix

$$
\begin{gathered}
\rho\left(t_{0}\right)=\rho_{S}\left(t_{0}\right) \otimes \rho_{B C}\left(t_{0}\right)=\rho_{S}\left(t_{0}\right) \otimes \rho_{B}\left(t_{0}\right) \otimes \rho_{C}\left(t_{0}\right) ; \\
\text { i.e., } \rho_{B C}\left(t_{0}\right)=\rho_{B}\left(t_{0}\right) \otimes \rho_{C}\left(t_{0}\right),
\end{gathered}
$$

to eliminate inhomogeneous term in Eq. (25). Returning back into the Schrödinger picture, the master equation takes the form

$$
\begin{aligned}
\frac{\partial}{\partial t} \rho_{S}(t)=-i\left[H_{a}(S), \rho_{S}(t)\right]- & \\
& \quad-\int_{t_{0}}^{t} d s \operatorname{Tr}_{B C}\left[\tilde{H}_{I}(0),\left[\tilde{H}_{I}(s-t), \rho_{S}(t) \rho_{B}\left(t_{0}\right) \rho_{C}\left(t_{0}\right)\right]\right] .
\end{aligned}
$$

Equation (25) can be transformed by writing the system-bath interaction Hamiltonian in a very general form

$$
H_{I}=\sum_{n} S_{n} E_{n},
$$

which reads in the interaction picture as

$$
\tilde{H}_{I}(t)=\sum_{n} \tilde{S}_{n}(t) \tilde{E}_{n}(t),
$$

where $S_{n}$ and $E_{n}$ are some system $S$ and environment $B, C$ operators only acting on the Hilbert space of the system and environment, respectively. As a consequence,

$$
\begin{aligned}
& \frac{\partial}{\partial t} \rho_{S}(t)=-i\left[H_{a}(S), \rho_{S}(t)\right]- \\
& \\
&-\sum_{n, m} \int_{0}^{t-t_{0}} d \tau\left[\tilde{S}_{n}(0) \tilde{S}_{m}(-\tau) \rho_{S}(t)-\tilde{S}_{m}(-\tau) \rho_{S}(t) \tilde{S}_{n}(0)\right] \times \\
& \quad \times \operatorname{Tr}_{B, C}\left\{\tilde{E}_{n}(0) \tilde{E}_{m}(-\tau) \rho_{B}\left(t_{0}\right) \rho_{C}\left(t_{0}\right)\right\}+
\end{aligned}
$$




$$
\begin{aligned}
& +\sum_{n, m} \int_{0}^{t-t_{0}} d \tau\left[\tilde{S}_{n}(0) \rho_{S}(t) \tilde{S}_{m}(-\tau)-\rho_{S}(t) \tilde{S}_{m}(-\tau) \tilde{S}_{n}(0)\right] \times \\
& \times \operatorname{Tr}_{B, C}\left\{\tilde{E}_{m}(-\tau) \tilde{E}_{n}(0) \rho_{B}\left(t_{0}\right) \rho_{C}\left(t_{0}\right)\right\} . \\
& \frac{\partial}{\partial t} \rho_{S}(t)=-i\left[H_{0}(S), \rho_{S}(t)\right]-i \frac{\lambda^{2}}{\omega}\left[\eta^{*} \mathrm{e}^{i \omega\left(t-t_{0}\right)} S^{-}+\eta \mathrm{e}^{-i \omega\left(t-t_{0}\right)} S^{+}, \rho_{S}(t)\right]- \\
& -\frac{\lambda^{2}}{V}\left\{\int_{0}^{t-t_{0}} d \tau\left[\tilde{S}^{-}(0) \tilde{S}^{+}(-\tau) \rho_{S}(t)-\tilde{S}^{+}(-\tau) \rho_{S}(t) \tilde{S}^{-}(0)\right] \times\right. \\
& \times \operatorname{Tr}_{B}\left\{\tilde{\hat{b}}^{+}(0) \tilde{\hat{b}}(-\tau) \rho_{B}\left(t_{0}\right)\right\}- \\
& -\int_{0}^{t-t_{0}} d \tau\left[\tilde{S}^{-}(0) \rho_{S}(t) \tilde{S}^{+}(-\tau)-\rho_{S}(t) \tilde{S}^{+}(-\tau) \tilde{S}^{-}(0)\right] \times \\
& \times \operatorname{Tr}_{B}\left\{\tilde{\hat{b}}(-\tau) \tilde{\hat{b}}^{+}(0) \rho_{B}\left(t_{0}\right)\right\}+ \\
& +\int_{0}^{t-t_{0}} d \tau\left[\tilde{S}^{+}(0) \tilde{S}^{-}(-\tau) \rho_{S}(t)-\tilde{S}^{-}(-\tau) \rho_{S}(t) \tilde{S}^{+}(0)\right] \times \\
& \times \operatorname{Tr}_{B}\left\{\tilde{\hat{b}}(0) \tilde{\hat{b}}^{+}(-\tau) \rho_{B}\left(t_{0}\right)\right\}- \\
& -\int_{0}^{t-t_{0}} d \tau\left[\tilde{S}^{+}(0) \rho_{S}(t) \tilde{S}^{-}(-\tau)-\rho_{S}(t) \tilde{S}^{-}(-\tau) \tilde{S}^{+}(0)\right] \times \\
& \times \operatorname{Tr}_{B}\left\{\tilde{\hat{b}}^{+}(-\tau) \tilde{\hat{b}}(0) \rho_{B}\left(t_{0}\right)\right\}+ \\
& +\int_{0}^{t-t_{0}} d \tau\left[\tilde{S}^{-}(0) \tilde{S}^{-}(-\tau) \rho_{S}(t)-\tilde{S}^{-}(-\tau) \rho_{S}(t) \tilde{S}^{-}(0)\right] \times \\
& \times \operatorname{Tr}_{B}\left\{\tilde{\hat{b}}^{+}(0) \tilde{\hat{b}}^{+}(-\tau) \rho_{B}\left(t_{0}\right)\right\}- \\
& -\int_{0}^{t-t_{0}} d \tau\left[\tilde{S}^{-}(0) \rho_{S}(t) \tilde{S}^{-}(-\tau)-\rho_{S}(t) \tilde{S}^{-}(-\tau) \tilde{S}^{-}(0)\right] \times \\
& \times \operatorname{Tr}_{B}\left\{\tilde{\hat{b}}^{+}(-\tau) \tilde{\hat{b}}^{+}(0) \rho_{B}\left(t_{0}\right)\right\}+ \\
& +\int_{0}^{t-t_{0}} d \tau\left[\tilde{S}^{+}(0) \tilde{S}^{+}(-\tau) \rho_{S}(t)-\tilde{S}^{+}(-\tau) \rho_{S}(t) \tilde{S}^{+}(0)\right] \times \\
& \times \operatorname{Tr}_{B}\left\{\tilde{\hat{b}}(0) \tilde{\hat{b}}(-\tau) \rho_{B}\left(t_{0}\right)\right\}- \\
& -\int_{0}^{t-t_{0}} d \tau\left[\tilde{S}^{+}(0) \rho_{S}(t) \tilde{S}^{+}(-\tau)-\rho_{S}(t) \tilde{S}^{+}(-\tau) \tilde{S}^{+}(0)\right] \times \\
& \left.\times \operatorname{Tr}_{B}\left\{\tilde{\hat{b}}(-\tau) \tilde{\hat{b}}(0) \rho_{B}\left(t_{0}\right)\right\}\right\}-
\end{aligned}
$$




$$
\begin{array}{r}
-\int_{0}^{t-t_{0}} d \tau \sum_{i=1}^{N} \sum_{\mathbf{k}} g_{i, \mathbf{k}}^{2}\left[\tilde{s}_{i}^{-}(0) \tilde{s}_{i}^{+}(-\tau) \rho_{S}(t)-\tilde{s}_{i}^{+}(-\tau) \rho_{S}(t) \tilde{s}_{i}^{-}(0)\right] \times \\
\times \operatorname{Tr}_{C}\left\{\tilde{c}_{i, \mathbf{k}}^{+}(0) \tilde{c}_{i, \mathbf{k}}(-\tau) \rho_{C}\left(t_{0}\right)\right\}+ \\
+\int_{0}^{t-t_{0}} d \tau \sum_{i=1}^{N} \sum_{\mathbf{k}} g_{i, \mathbf{k}}^{2}\left[\tilde{s}_{i}^{-}(0) \rho_{S}(t) \tilde{s}_{i}^{+}(-\tau)-\rho_{S}(t) \tilde{s}_{i}^{+}(-\tau) \tilde{s}_{i}^{-}(0)\right] \times \\
\times \operatorname{Tr}_{C}\left\{\tilde{c}_{i, \mathbf{k}}(-\tau) \tilde{c}_{i, \mathbf{k}}^{+}(0) \rho_{C}\left(t_{0}\right)\right\}- \\
-\int_{0}^{t-t_{0}} d \tau \sum_{i=1}^{N} \sum_{\mathbf{k}} g_{i, \mathbf{k}}^{2}\left[\tilde{s}_{i}^{+}(0) \tilde{s}_{i}^{-}(-\tau) \rho_{S}(t)-\tilde{s}_{i}^{-}(-\tau) \rho_{S}(t) \tilde{s}_{i}^{+}(0)\right] \times \\
\times \operatorname{Tr}_{C}\left\{\tilde{c}_{i, \mathbf{k}}(0) \tilde{c}_{i, \mathbf{k}}^{+}(-\tau) \rho_{C}\left(t_{0}\right)\right\}+ \\
+\int_{0}^{t-t_{0}} d \tau \sum_{i=1}^{N} \sum_{\mathbf{k}} g_{i, \mathbf{k}}^{2}\left[\tilde{s}_{i}^{+}(0) \rho_{S}(t) \tilde{s}_{i}^{-}(-\tau)-\rho_{S}(t) \tilde{s}_{i}^{-}(-\tau) \tilde{s}_{i}^{+}(0)\right] \times \\
\times \operatorname{Tr}_{C}\left\{\tilde{c}_{i, \mathbf{k}}^{+}(-\tau) \tilde{c}_{i, \mathbf{k}}(0) \rho_{C}\left(t_{0}\right)\right\}
\end{array}
$$

where

$$
\hat{b}^{+}=b^{+}+\sqrt{V} \frac{\lambda}{\omega} \eta^{*}, \quad b=b+\sqrt{V} \frac{\lambda}{\omega} \eta
$$

are shifted Bose operators. Time dependence of the operators $\tilde{S}^{z}(\tau), \tilde{S}^{+}(\tau)$ and $\tilde{S}^{-}(\tau)$ in (32) can be found explicitly by means of the following relations derived in $[3,4]$ :

$$
\begin{gathered}
\mathrm{e}^{i\left(b_{1} S^{z}+b_{2} S^{-}+b_{2}^{*} S^{+}\right)} S^{z} \mathrm{e}^{-i\left(b_{1} S^{z}+b_{2} S^{-}+b_{2}^{*} S^{+}\right)}=\frac{\left|b_{1}\right|^{2}+4\left|b_{2}\right|^{2} \cos b}{b^{2}} S^{z}+ \\
+\left\{(1-\cos b) \frac{b_{1} b_{2}}{b^{2}}+i \frac{b_{2}}{b} \sin b\right\} S^{-}+ \\
+\left\{(1-\cos b) \frac{b_{1} b_{2}^{*}}{b^{2}}+i \frac{b_{2}^{*}}{b} \sin b\right\} S^{+}, \\
\mathrm{e}^{i\left(b_{1} S^{z}+b_{2} S^{-}+b_{2}^{*} S^{+}\right)} S^{-} \mathrm{e}^{-i\left(b_{1} S^{z}+b_{2} S^{-}+b_{2}^{*} S^{+}\right)}= \\
=\left\{2(1-\cos b) \frac{b_{1} b_{2}^{*}}{b^{2}}+2 i \frac{b_{2}^{*}}{b} \sin b\right\} S^{z}+ \\
+\left\{\frac{\left|b_{1}\right|^{2} \cos b+2\left|b_{2}\right|^{2}(1+\cos b)}{b^{2}}-i \frac{b_{1}}{b} \sin b\right\} S^{-}+ \\
+2 \frac{\left(b_{2}^{*}\right)^{2}}{b^{2}}(1-\cos b) S^{+}
\end{gathered}
$$




$$
\begin{gathered}
\mathrm{e}^{i\left(b_{1} S^{z}+b_{2} S^{-}+b_{2}^{*} S^{+}\right)} S^{+} \mathrm{e}^{-i\left(b_{1} S^{z}+b_{2} S^{-}+b_{2}^{*} S^{+}\right)}= \\
=\left\{2(1-\cos b) \frac{b_{1} b_{2}}{b^{2}}-2 i \frac{b_{2}}{b} \sin b\right\} S^{z}+ \\
+\left\{\frac{\left|b_{1}\right|^{2} \cos b+2\left|b_{2}\right|^{2}(1+\cos b)}{b^{2}}-i \frac{b_{1}}{b} \sin b\right\} S^{-}+ \\
+2 \frac{b_{2}^{2}}{b^{2}}(1-\cos b) S^{+}, \\
b^{2}=\left|b_{1}\right|^{2}+4\left|b_{2}\right|^{2}, \quad b_{1}=\epsilon \tau, \quad b_{2}=-2 \frac{\lambda^{2}}{\omega} \eta^{*} \tau
\end{gathered}
$$

and all the thermodynamically equilibrium correlation functions of the kind

$$
\begin{gathered}
\operatorname{Tr}_{B}\left\{\tilde{\hat{b}}^{+}(0) \tilde{\hat{b}}(-\tau) \rho_{B}\left(t_{0}\right)\right\}, \quad \operatorname{Tr}_{B}\left\{\tilde{\hat{b}}^{+}(0) \tilde{\hat{b}}^{+}(-\tau) \rho_{B}\left(t_{0}\right)\right\}, \\
\operatorname{Tr}_{C}\left\{\tilde{c}_{i, \mathbf{k}}^{+}(0) \tilde{c}_{i, \mathbf{k}}(-\tau) \rho_{C}\left(t_{0}\right)\right\}
\end{gathered}
$$

belonging to subsystems $B$ and $C$ can be calculated explicitly too, so that all the integrations over $\tau$ can be carried out analytically.

\section{DYNAMICS OF EXCITED MAGNETIC MOMENTS}

As to the initial condition (26), $\rho_{B}\left(t_{0}=0\right)$ and $\rho_{C}\left(t_{0}=0\right)$ can be, formally, an arbitrary density operator pertaining to the subsystems $B$ and $C$. It is justified, for example, to take them in the form of the thermodynamically equilibrium density operators

$$
\begin{aligned}
& \rho_{C}\left(t_{0}\right)=\frac{\mathrm{e}^{-\beta H_{0}(C)}}{\operatorname{Tr}_{C}\left(\mathrm{e}^{-\beta H_{0}(C)}\right)}, \\
& \rho_{B}\left(t_{0}\right)=\frac{\mathrm{e}^{-\beta H_{0}(B)}}{\operatorname{Tr}_{B}\left(\mathrm{e}^{-\beta H_{0}(B)}\right)}, \quad \text { or } \\
& \rho_{B}\left(t_{0}\right)=\frac{\mathrm{e}^{-\beta H_{a}(B)}}{\operatorname{Tr}_{B}\left(\mathrm{e}^{-\beta H_{a}(B)}\right)} .
\end{aligned}
$$

This initial condition corresponds to the situation when all subsystems, $S, B$ and $C$, were originally in thermodynamic equilibrium characterized by the common temperature $\theta=1 / \beta$, and then the subsystem $S$ was excited to some initial state $\rho_{S}\left(t_{0}\right)$, while the environment $B, C$ remained in its initial equilibrium state $\rho_{B}\left(t_{0}\right) \otimes \rho_{C}\left(t_{0}\right)$ due to its significant inertia. The choice (39) corresponds to the original Bose mode $b$ being in the initial state of thermal equilibrium, while the choice (40) describes thermally equilibrium Bose field (36) shifted due to the interaction with magnetic moments. 
The last four lines in Eq. (32) are rather trivial. They describe the process of steady exponential relaxation of excited magnetic moments to the state of thermal equilibrium due to interaction with the heat bath $C$ and can be handled as usual (see, for example, [5]).

The second term on the right-hand side of Eq. (32) is much more interesting because it shows that for $T<T_{c}$, i.e., when $\eta \neq 0$, a kind of an external pumping field arises in the $x-y$ plane. This field drives excited magnetic moments forcing them to flip fro and back along the $z$-axis with the so-called Rabi frequency:

$$
\Omega=\sqrt{(\epsilon-\omega)^{2}+\left(\frac{\lambda^{2}|\eta|}{\omega}\right)^{2}} .
$$

Of course, this field is transient in reality and this driving cannot last forever, because the Bose field mode $b$ is always damped to some extent due to the cavity imperfections. This damping may be accounted for by inclusion of the damping factor $\mathrm{e}^{-\tau / \tau_{b}}$ into the correspondent correlation functions (37). Here we intentionally omitted an additional interaction of the field mode $b$ with its own heat bath.

The lines from 6th to 9th containing anomalous bosonic correlation functions may only be present in Eq. (32) if the initial density operator $\rho_{B}\left(t_{0}\right)$ was chosen in the form (39) and only for $T<T_{c}$ when $\eta \neq 0$. But even in this case all these terms are proportional to $\frac{\lambda^{4}}{\omega^{2}(\epsilon+\omega)}|\eta|^{2}$ and, in addition, they are fast oscillating terms and can be averaged out.

The lines from 2 nd to 5 th containing normal bosonic correlation functions represent usual behavior of magnetic moments under the influence of thermal Bose field mode $b$ for both choices (39), (40) of $\rho_{B}\left(t_{0}\right)$ if $T>T_{c}$ and $\eta=0$. The same situation persists even for $T<T_{c}$ for the choice (40). These lines are proportional to $1 / V$ so that the contribution of these terms is not large in the thermodynamic limit. But for the choice (39) and $T<T_{c}, \eta \neq 0$ a new factor $V \frac{\lambda^{2}}{\omega^{2}}|\eta|^{2}$ arises from the bosonic correlation functions which compensates this $1 / V$ multiplier and makes the contribution of these terms more or less tangible.

Also, as it follows from relations (33)-(35), the time dependence of the operators $\tilde{S}^{ \pm}(\tau)$ is altered for $T<T_{c}, \eta \neq 0$. But new additional terms in (33)(35) will be proportional to $\left(\frac{\lambda^{2}}{\omega}|\eta|^{2}\right)^{2}$ so that their effect is not very pronounced.

Therefore, the most pronounced, and possibly experimentally observable, phenomenon would be an appearance of effective transient driving oscillating field resulting in deviation of the excited magnetic moments relaxation from the exponential law in the form of Rabi oscillation superimposed onto their usual relaxational behavior. 


\section{SUMMARY}

It was shown that, when it comes to investigating non-equilibrium properties of a physical system, it may be advantageous to decompose the original total Hamiltonian of the system into two parts by means of the identity transformation in order to capture some specific features of the system dynamics in a more transparent way. Of course, from the strictly mathematical point of view, any formally precise master equation must provide one with correct description of the dynamics of the system and its subsystems notwithstanding any partition of the total system Hamiltonian into the so-called "free" and "interaction" Hamiltonians. Unfortunately, precise solutions of master equations are almost never available in practice. To overcome this difficulty, one has to deal with some approximations to the original master equation and the outcome of this approximations is dependent significantly upon the above-mentioned partition of the original Hamiltonian. The way of partitioning is not formal and unique, so that one has to rely on some a priori physical ideas and understanding. Here we presented one possible way of such a partitioning based on the ideas of the approximating Hamiltonian method, which has already proved itself useful in the studies of equilibrium properties of various many-particle systems.

\section{ACKNOWLEDGEMENTS}

This work is supported by the RSF under a grant 14-50-00005.

\section{REFERENCES}

1. N.N. Bogolyubov (Jr.), J.G. Brankov, V.A. Zagrebnov, A.M. Kurbatov, N.S. Tonchev: The Approximating Hamiltonian Method in Statistical Physics (Publishing House of the Bulgarian Academy of Sciences, Sofia, 1981).

2. H.-P. Breuer, F. Petruccione: The Theory of Open Quantum Systems (Oxford University Press, 2002).

3. G.M. Ershov: in Proc. of the All-Union conference on paramagnetic resonance, Kazan, 1969, p.264.

4. U.Kh. Kopvillem, S.V. Prants: Polarization Echo, Moscow, Nauka, 1985, p.20.

5. H. Carmichael: An Open Systems Approach to Quantum Optics (Volume m18 of Lecture Notes in Physics. Springer-Verlag, Berlin, 1993).

Received on February 2, 2016. 


\section{Редактор Е. И. Кравченко}

Подписано в печать 18.02.2016.

Формат $60 \times 90 / 16$. Бумага офсетная. Печать офсетная.

Усл. печ. л. 0,87. Уч.-изд. л. 1,2. Тираж 225 экз. Заказ № 58755.

Издательский отдел Объединенного института ядерных исследований 141980 , г. Дубна, Московская обл., ул. Жолио-Кюри, 6.

E-mail: publish@jinr.ru www.jinr.ru/publish/ 